\title{
Simultaneous Feedback Edge Set: A Parameterized Perspective*
}

\author{
Akanksha Agrawal ${ }^{1}$, Fahad Panolan ${ }^{2}$, Saket Saurabh ${ }^{3}$, and \\ Meirav Zehavi ${ }^{4}$
}

1 Department of Informatics, University of Bergen, Norway

akanksha.agrawal@uib.no

2 Department of Informatics, University of Bergen, Norway

fahad.panolan@uib.no

3 Department of Informatics, University of Bergen, Norway; and

The Institute of Mathematical Sciences, HBNI, Chennai, India

saket@imsc.res.in

4 Department of Informatics, University of Bergen, Norway

zehavimeirav@gmail.com

\begin{abstract}
In a recent article Agrawal et al. (STACS 2016) studied a simultaneous variant of the classic Feedback Vertex Set problem, called Simultaneous Feedback Vertex Set (Sim-FVS). In this problem the input is an $n$-vertex graph $G$, an integer $k$ and a coloring function col : $E(G) \rightarrow 2^{[\alpha]}$, and the objective is to check whether there exists a vertex subset $S$ of cardinality at most $k$ in $G$ such that for all $i \in[\alpha], G_{i}-S$ is acyclic. Here, $G_{i}=(V(G),\{e \in E(G) \mid$ $i \in \operatorname{col}(e)\})$ and $[\alpha]=\{1, \ldots, \alpha\}$. In this paper we consider the edge variant of the problem, namely, Simultaneous Feedback Edge Set (Sim-FES). In this problem, the input is same as the input of SIm-FVS and the objective is to check whether there is an edge subset $S$ of cardinality at most $k$ in $G$ such that for all $i \in[\alpha], G_{i}-S$ is acyclic. Unlike the vertex variant of the problem, when $\alpha=1$, the problem is equivalent to finding a maximal spanning forest and hence it is polynomial time solvable. We show that for $\alpha=3$ Sim-FES is NP-hard by giving a reduction from VERTEX COVER on cubic-graphs. The same reduction shows that the problem does not admit an algorithm of running time $\mathcal{O}\left(2^{o(k)} n^{\mathcal{O}(1)}\right)$ unless ETH fails. This hardness result is complimented by an FPT algorithm for SIM-FES running in time $\mathcal{O}\left(2^{\omega k \alpha+\alpha \log k} n^{\mathcal{O}(1)}\right)$, where $\omega$ is the exponent in the running time of matrix multiplication. The same algorithm gives a polynomial time algorithm for the case when $\alpha=2$. We also give a kernel for SIM-FES with $(k \alpha)^{\mathcal{O}(\alpha)}$ vertices. Finally, we consider the problem Maximum Simultaneous ACyCliC SuBGRAPH. Here, the input is a graph $G$, an integer $q$ and, a coloring function col : $E(G) \rightarrow 2^{[\alpha]}$. The question is whether there is a edge subset $F$ of cardinality at least $q$ in $G$ such that for all $i \in[\alpha], G\left[F_{i}\right]$ is acyclic. Here, $F_{i}=\{e \in F \mid i \in \operatorname{col}(e)\}$. We give an FPT algorithm for Maximum Simultaneous Acyclic Subgraph running in time $\mathcal{O}\left(2^{\omega q \alpha} n^{\mathcal{O}(1)}\right)$. All our algorithms are based on parameterized version of the MATROID PARITY problem.
\end{abstract}

1998 ACM Subject Classification G.2.2 Graph Algorithms, I.1.2 Analysis of Algorithms

Keywords and phrases parameterized complexity, feedback edge set, $\alpha$-matroid parity

Digital Object Identifier 10.4230/LIPIcs.ISAAC.2016.5

\footnotetext{
* The research leading to these results has received funding from the European Research Council (ERC) via grants Rigorous Theory of Preprocessing, reference 267959 and PARAPPROX, reference 306992.
}

(c) (i) Akanksha Agrawal, Fahad Panolan, Saket Saurabh, and Meirav Zehavi;

27th International Symposium on Algorithms and Computation (ISAAC 2016).

Editor: Seok-Hee Hong; Article No. 5; pp. 5:1-5:13

Leibniz International Proceedings in Informatics

LI I ICS Schloss Dagstuhl - Leibniz-Zentrum für Informatik, Dagstuhl Publishing, Germany 


\section{Introduction}

Deleting at most $k$ vertices or edges from a given graph $G$, so that the resulting graph belongs to a particular family of graphs $(\mathcal{F})$, is an important research direction in the fields of graph algorithms and parameterized complexity. For a family of graphs $\mathcal{F}$, given a graph $G$ and an integer $k$, the $\mathcal{F}$-DELETION (EDGE $\mathcal{F}$-DELETION) problem asks whether we can delete at most $k$ vertices (edges) in $G$ so that the resulting graph belongs to $\mathcal{F}$. The $\mathcal{F}$-DELETION (EdGE $\mathcal{F}$-DELETION) problems generalize many of the NP-hard problems like Vertex Cover, Feedback vertex set, Odd cycle transversal, Edge Bipartization, etc. Inspired by applications, Cai and Ye introduced variants of $\mathcal{F}$-DELETION (EDGE $\mathcal{F}$ DELETION) problems on edge colored graph [7]. Edge colored graphs are studied in graph theory with respect to various problems like Monochromatic and HeTERochromatiC Subgraphs [15], Alternating Paths [6, 8, 20], Homomorphism in edge-colored graphs [3], Graph PARTitioning in 2-edge colored graphs [5] etc. One of the natural generalization to the classic $\mathcal{F}$-DELETION (EDGE $\mathcal{F}$-DELETION) problems on edge colored graphs is the following. Given a graph $G$ with a coloring function col : $E(G) \rightarrow 2^{[\alpha]}$, and an integer $k$, we want to delete a set $S$ of at most $k$ edges/vertices in $G$ so that for each $i \in[\alpha], G_{i}-S$ belongs to $\mathcal{F}$. Here, $G_{i}$ is the graph with vertex set $V(G)$ and edge set as $\{e \in E(G) \mid i \in \operatorname{col}(e)\}$. These problems are also called simultaneous variant of $\mathcal{F}$-DELETION (EDGE $\mathcal{F}$-DELETION).

Cai and Ye studied the Dually Connected Induced subgraph and Dual Separator on 2-edge colored graphs [7]. Agrawal et al. [1] studied a simultaneous variant of FEEDBACK Vertex Set problem, called Simultaneous Feedback Vertex Set, in the realm of parameterized complexity. Here, the input is a graph $G$, an integer $k$, and a coloring function col : $E(G) \rightarrow 2^{[\alpha]}$ and the objective is to check whether there is a set $S$ of at most $k$ vertices in $G$ such that for all $i \in[\alpha], G_{i}-S$ is acyclic. Here, $G_{i}=(V(G),\{e \in E(G) \mid i \in \operatorname{col}(e)\})$. In this paper we consider the edge variant of the problem, namely, Simultaneous FEEDbaCK EDGE SET, in the realm of parameterized complexity.

In the Parameterized Complexity paradigm the main objective is to design an algorithm with running time $f(\mu) \cdot n^{\mathcal{O}(1)}$, where $\mu$ is the parameter associated with the input, $n$ is the size of the input and $f(\cdot)$ is some computable function whose value depends only on $\mu$. A problem which admits such an algorithm is said to be fixed parameter tractable parameterized by $\mu$. Typically, for edge/vertex deletion problems one of the natural parameter that is associated with the input is the size of the solution we are looking for. Another objective in parameterized complexity is to design polynomial time pre-processing routines that reduces the size of the input as much as possible. The notion of such a pre-processing routine is captured by kernelization algorithms. The kernelization algorithm for a parameterized problem $Q$ takes as input an instance $(I, k)$ of $Q$, runs in polynomial time and returns an equivalent instance $\left(I^{\prime}, k^{\prime}\right)$ of $Q$. Moreover, the size of the instance $\left(I^{\prime}, k^{\prime}\right)$ returned by the kernelization algorithm is bounded by $g(k)$, where $g(\cdot)$ is some computable function whose value depends only on $k$. If $g(\cdot)$ is polynomial in $k$, then the problem $Q$ is said to admit a polynomial kernel. The instance returned by the kernelization is referred to as a kernel or a reduced instance. We refer the readers to the recent book of Cygan et al. [9] for a more detailed overview of parameterized complexity and kernelization.

A feedback edge set in a graph $G$ is $S \subseteq E(G)$ such that $G-S$ is a forest. For a graph $G$ with a coloring function col : $E(G) \rightarrow 2^{[\alpha]}$, simultaneous feedback edge set is a subset $S \subseteq E(G)$ such that $G_{i}-S$ is a forest for all $i \in[\alpha]$. Here, $G_{i}=\left(V(G), E_{i}\right)$, where $E_{i}=\{e \in E(G) \mid i \in \operatorname{col}(e)\}$. Formally, the problem is stated below. 
Simultaneous Feedback Edge Set (Sim-FES)

Parameter: $k, \alpha$

Input: An $n$-vertex graph $G$, an integer $k$ and a coloring function col : $E(G) \rightarrow 2^{[\alpha]}$

Question: Is there a simultaneous feedback edge set of cardinality at most $k$ in $G$

FEEDBACK VERTEX SET (FVS) is one of the classic NP-complete [13] problems and has been extensively studied from all the algorithmic paradigms that are meant for coping with NP-hardness, such as approximation algorithms, parameterized complexity and moderately exponential time algorithms. The problem admits a factor 2-approximation algorithm [4], an exact algorithm with running time $\mathcal{O}\left(1.7217^{n} n^{\mathcal{O}(1)}\right)[12]$, a deterministic parameterized algorithm running in $\mathcal{O}\left(3.619^{k} n^{\mathcal{O}(1)}\right)$ time [16], a randomized algorithm running in $\mathcal{O}\left(3^{k} n^{\mathcal{O}(1)}\right)$ time [10], and a kernel with $\mathcal{O}\left(k^{2}\right)$ vertices [24]. Agrawal et al. [1] studied Simultaneous FEEDBACK VERTEX SET (SIM-FVS) and gave an FPT algorithm running in time $2^{\mathcal{O}(\alpha k)} n^{\mathcal{O}(1)}$ and a kernel of size $\mathcal{O}\left(\alpha k^{3(\alpha+1)}\right)$. Finally, unlike the FVS problem, Sim-FES is polynomial time solvable when $\alpha=1$, because it is equivalent to finding maximal spanning forest.

Our results and approach. In Section 3 we design an FPT algorithm for SIM-FES by reducing to $\alpha$-MATROID PARITY on direct sum of elongated co-graphic matroids of $G_{i}$, $i \in[\alpha]$ (see Section 2 for definitions related to matroids). This algorithm runs in time $\mathcal{O}\left(2^{\omega k \alpha+\alpha \log k} n^{\mathcal{O}(1)}\right)$. Unlike the vertex counterpart, we show that for $\alpha=2$ (2-edge colored graphs) Sim-FES is polynomial time solvable. This follows from the polynomial time algorithm for the MATROID PARITY problem. In Section 4 we show that for $\alpha=3$, Sim-FES is NP-hard. Towards this, we give a reduction from the VERTEx Cover in cubic graphs which is known to be NP-hard [22]. Furthermore, the same reduction shows that the problem cannot be solved in $2^{o(k)} n^{\mathcal{O}(1)}$ time unless Exponential Time Hypothesis (ETH) fails [14]. We complement our FPT algorithms by showing that SIM-FES is W[1]-hard when parameterized by the solution size $k$ (Section 5 ). When $\alpha=\mathcal{O}(|V(G)|)$, we give a parameter preserving reduction from the HitTing SET problem, a well known W[2]-hard problem parameterized by the solution size [9]. However, SIM-FES remains W[1]-hard even when $\alpha=\mathcal{O}(\log (|V(G)|))$. We show this by giving a parameter preserving reduction from PARTitioned Hitting SET problem, a variant of the Hitting SET problem, defined in [1]. In [1], PARTitioned Hitting SET was shown to be W[1]-hard parameterized by the solution size. In Section 6 we give a kernel with $\mathcal{O}\left((k \alpha)^{\mathcal{O}(\alpha)}\right)$ vertices. Towards this we apply some of the standard preprocessing rules for obtaining kernel for FEEDBACK VERTEx SET and use the approach similar to the one developed for designing kernelization algorithm for SIM-FVS [1]. In Section 7 we give an FPT algorithm for the problem, when parameterized by the dual parameter. Formally, this problem is defined as follows.

Maximum Simultaneous Acyclic Subgraph (MaX-Sim-Subgraph) Parameter: $q$

Input: An $n$-vertex graph $G$, a positive integer $q$ and a function col : $E(G) \rightarrow 2^{[\alpha]}$.

Question: Is there a subset $F \subseteq E(G)$ such that $|F| \geq q$ and for all $i \in[\alpha], G\left[F \cap E\left(G_{i}\right)\right]$ is acyclic?

For solving MAX-Sim-SubGraPH we reduce it to an equivalent instance of the $\alpha$-MATROID PARITY problem. As an immediate corollary we get an exact algorithm for SIM-FES running in time $\mathcal{O}\left(2^{\omega n \alpha^{2}} n^{\mathcal{O}(1)}\right)$.

\section{Preliminaries}

We denote the set of natural numbers by $\mathbb{N}$. For $n \in \mathbb{N}$, by $[n]$ we denote the set $\{1, \ldots, n\}$. For a set $X$, by $2^{X}$ we denote the set of all subsets of $X$. We use the term ground set/ universe 
to distinguish a set from its subsets. We will use $\omega$ to denote the exponent in the running time of matrix multiplication, the current best known bound for which is $\omega<2.373$ [25].

Graphs. We use the term graph to denote undirected graph. For a graph $G$, by $V(G)$ and $E(G)$ we denote its vertex set and edge set, respectively. We will be considering finite graphs possibly having loops and multi-edges. In the following, let $G$ be a graph and let $H$ be a subgraph of $G$. By $d_{H}(v)$, we denote the degree of the vertex $v$ in $H$, i.e, the number of edges in $\mathrm{H}$ which are incident with $v$. A self-loop at a vertex $v$ contributes 2 to the degree of $v$. For any non-empty subset $W \subseteq V(G)$, the subgraphs of $G$ induced by $W, V(G) \backslash W$ are denoted by $G[W]$ and $G-W$ respectively. Similarly, for $F \subseteq E(G)$, the subgraph of $G$ induced by $F$ is denoted by $G[F]$; its vertex set is $V(G)$ and its edge set is $F$. For $F \subseteq E(G)$, by $G-F$ we denote the graph obtained by deleting the edges in $F$. We use the convention that a double edge and a self-loop is a cycle. An $\alpha$-edge colored graph is a graph $G$ with a color function col : $E(G) \rightarrow 2^{[\alpha]}$. By $G_{i}$ we will denote the color $i$ (or $i$-color) graph of $G$, where $V\left(G_{i}\right)=V(G)$ and $E\left(G_{i}\right)=\{e \in E(G) \mid i \in \operatorname{col}(e)\}$. For an $\alpha$-edge colored graph $G$, the total degree of a vertex $v$ is $\sum_{i=1}^{\alpha} d_{G_{i}}(v)$. We refer the reader to [11] for details on standard graph theoretic notations and terminologies.

Matroids and Representable Matroids. A pair $M=(E, \mathcal{I})$, where $E$ is a ground set and $\mathcal{I}$ is a family of subsets (called independent sets) of $E$, is a matroid if it satisfies the following conditions: (I1) $\phi \in \mathcal{I}$. (I2) If $A^{\prime} \subseteq A$ and $A \in \mathcal{I}$ then $A^{\prime} \in \mathcal{I}$. (I3) If $A, B \in \mathcal{I}$ and $|A|<|B|$, then there is $e \in(B \backslash A)$ such that $A \cup\{e\} \in \mathcal{I}$. We refer the reader to [23] for more details.

Let $A$ be a matrix over an arbitrary field $\mathbb{F}$ and let $E$ be the set of columns of $A$. For $A$, we define matroid $M=(E, \mathcal{I})$ as follows. A set $X \subseteq E$ is independent (that is $X \in \mathcal{I}$ ) if the corresponding columns are linearly independent over $\mathbb{F}$. The matroids that can be defined by such a construction are called linear matroids, and if a matroid can be defined by a matrix $A$ over a field $\mathbb{F}$, then we say that the matroid is representable over $\mathbb{F}$. A matroid $M=(E, \mathcal{I})$ is called representable or linear if it is representable over some field $\mathbb{F}$.

Direct Sum of Matroids. Let $M_{1}=\left(E_{1}, \mathcal{I}_{1}\right), M_{2}=\left(E_{2}, \mathcal{I}_{2}\right), \ldots, M_{t}=\left(E_{t}, \mathcal{I}_{t}\right)$ be $t$ matroids with $E_{i} \cap E_{j}=\emptyset$ for all $1 \leq i \neq j \leq t$. The direct sum $M_{1} \oplus \cdots \oplus M_{t}$ is a matroid $M=(E, \mathcal{I})$ with $E:=\bigcup_{i=1}^{t} E_{i}$ and $X \subseteq E$ is independent if and only if $X \cap E_{i} \in \mathcal{I}_{i}$ for all $i \in[t]$. Let $A_{i}$ be the representation matrix of $M_{i}=\left(E_{i}, \mathcal{I}_{i}\right)$ over field $\mathbb{F}$. Then, a representation matrix of $M_{1} \oplus \cdots \oplus M_{t}$ over $\mathbb{F}$ can be found in polynomial time [21, 23].

Uniform Matroid. A pair $M=(E, \mathcal{I})$ over an $n$-element ground set $E$, is called a uniform matroid if the family of independent sets is given by $\mathcal{I}=\{A \subseteq E|| A \mid \leq k\}$, where $k$ is some constant. This matroid is also denoted as $U_{n, k}$.

- Proposition 2.1 ([9, 23]). Uniform matroid $U_{n, k}$ is representable over any field of size strictly more than $n$ and such a representation can be found in time polynomial in $n$.

Graphic and Cographic Matroid. Given a graph $G$, the graphic matroid $M=(E, \mathcal{I})$ is defined by taking the edge set $E(G)$ as universe and $F \subseteq E(G)$ is in $\mathcal{I}$ if and only if $G[F]$ is a forest. Let $G$ be a graph and $\eta$ be the number of components in $G$. The co-graphic matroid $M=(E, \mathcal{I})$ of $G$ is defined by taking the the edge set $E(G)$ as universe and $F \subseteq E(G)$ is in $\mathcal{I}$ if and only if the number of connected components in $G-F$ is $\eta$.

- Proposition 2.2 ([23]). Graphic and co-graphic matroids are representable over any field of size $\geq 2$ and such a representation can be found in time polynomial in the size of the graph. 
Elongation of Matroid. Let $M=(E, \mathcal{I})$ be a matroid and $k$ be an integer such that $\operatorname{rank}(M) \leq k \leq|E|$. A $k$-elongation matroid $M_{k}$ of $M$ is a matroid with the universe as $E$ and $S \subseteq E$ is a basis of $M_{k}$ if and only if, it contains a basis of $M$ and $|S|=k$. Observe that the rank of the matroid $M_{k}$ is $k$.

- Proposition 2.3 ([18]). Let $M$ be a linear matroid of rank $r$, over a ground set of size $n$, which is representable over a field $\mathbb{F}$. Given a number $\ell \geq r$, we can compute a representation of the $\ell$-elongation of $M$, over the field $\mathbb{F}(X)$ in $\mathcal{O}($ nr $\ell)$ field operations over $\mathbb{F}$.

$\boldsymbol{\alpha}$-Matroid Parity. In our algorithms we use a known algorithm for $\alpha$-MATROID PARITY. Below we define $\alpha$-MATROID PARITY problem formally and state its algorithmic result.

$\alpha$-MATROID PARITY

Parameter: $\alpha, q$

Input: A representation $A_{M}$ of a linear matroid $M=(E, \mathcal{I})$, a partition $\mathcal{P}$ of $E$ into blocks of size $\alpha$ and a positive integer $q$.

Question: Does there exist an independent set which is a union of $q$ blocks?

- Proposition $2.4([18,21])$. $\alpha$-Matroid Parity can be solved in $\mathcal{O}\left(2^{\omega q \alpha}\left\|A_{M}\right\|{ }^{\mathcal{O}(1)}\right)$ time.

\section{FPT Algorithm for Simultaneous Feedback Edge Set}

In this section we design an algorithm for SIM-FES by giving a reduction to $\alpha$-MATROID PARITY on the direct sum of elongated co-graphic matroids associated with graphs restricted to different color classes.

We describe our algorithm, Algo-SimFES, for Sim-FES. Let $\left(G, k\right.$, col : $\left.E(G) \rightarrow 2^{[\alpha]}\right)$ be an input instance to SIM-FES. Recall that for $i \in[\alpha], G_{i}$ is the graph with vertex set as $V(G)$ and edge set as $E\left(G_{i}\right)=\{e \in E(G) \mid i \in \operatorname{col}(e)\}$. Let $n=|V(G)|$. Note that $n=\left|V\left(G_{i}\right)\right|$ for all $i \in[\alpha]$. Let $\eta_{i}$ be the number of connected components in $G_{i}$. To make $G_{i}$ acyclic we need to delete at least $\left|E\left(G_{i}\right)\right|-n+\eta_{i}$ edges from $G_{i}$. Therefore, if there is $i \in[\alpha]$ such that $\left|E\left(G_{i}\right)\right|-n+\eta_{i}>k$, then Algo-SimFES returns No. We let $k_{i}=\left|E\left(G_{i}\right)\right|-n+\eta_{i}$. Observe that for $i \in[\alpha], 0 \leq k_{i} \leq k$. We need to delete at least $k_{i}$ edges from $E\left(G_{i}\right)$ to make $G_{i}$ acyclic. Therefore, the algorithm Alg-SimFES for each $i \in[\alpha]$, guesses $k_{i}^{\prime}$, where $k_{i} \leq k_{i}^{\prime} \leq k$ and computes a solution $S$ of Sim-FES such that $\left|S \cap E\left(G_{i}\right)\right|=k_{i}^{\prime}$. Let $M_{i}=\left(E_{i}, \mathcal{I}_{i}\right)$ be the $k_{i}^{\prime}$-elongation of the co-graphic matroid associated with $G_{i}$.

- Proposition $3.1\left(*^{1}\right)$. Let $G$ be a graph with $\eta$ connected components and $M$ be an $r$ elongation of the co-graphic matroid associated with $G$, where $r \geq|E(G)|-|V(G)|+\eta$. Then $B \subseteq E(G)$ is a basis of $M$ if and only if the subgraph $G-B$ is acyclic and $|B|=r$.

By Proposition 3.1, for any basis $F_{i}$ in $M_{i}, G_{i}-F_{i}$ is acyclic. Therefore, our objective is to compute $F \subseteq E(G)$ such that $|F|=k$ and the elements of $F$ restricted to the elements of $M_{i}$ form a basis for all $i \in[\alpha]$. For this we will construct an instance of $\alpha$-Matroid PARity as follows. For each $e \in E(G)$ and $i \in \operatorname{col}(e)$, we use $e^{i}$ to denote the corresponding element in $M_{i}$. For each $e \in E(G)$, by Original $(e)$ we denote the set of elements $\left\{e^{j} \mid j \in \operatorname{col}(e)\right\}$. For each edge $e \in E(G)$, we define Fake $(e)=\left\{e^{j} \mid j \in[\alpha]-\operatorname{col}(e)\right\}$. Finally, for each edge $e \in E(G)$, by Copies $(e)$ we denote the set Original $(e) \cup$ Fake $(e)$. Let Fake $(G)=\bigcup_{e \in E(G)} \operatorname{Fake}(e)$. Furthermore, let $\tau=|\operatorname{Fake}(G)|=\sum_{e \in E(G)}|\operatorname{Fake}(e)|$ and

\footnotetext{
1 Proofs of results marked with $(*)$ can be found in the full version of the paper [2].
} 


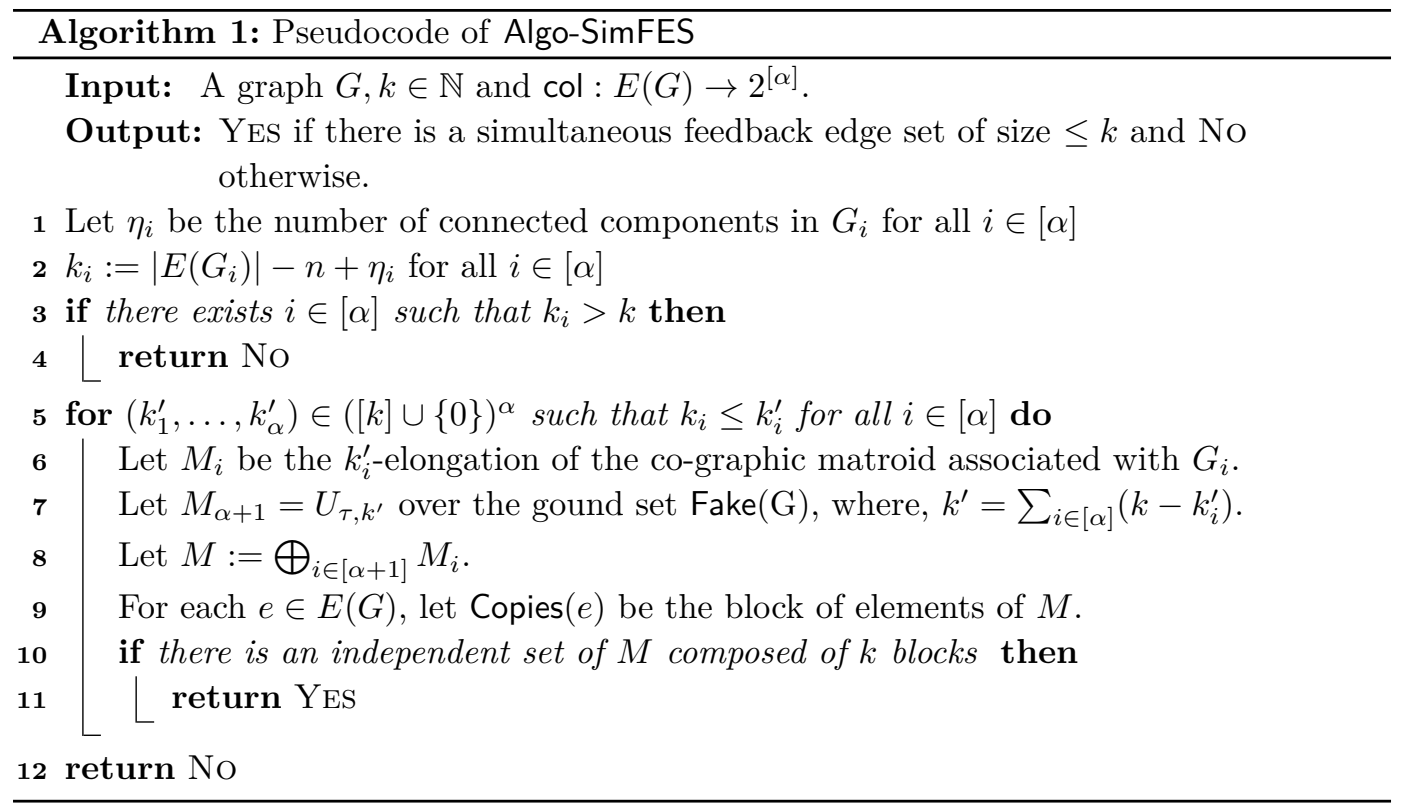

$k^{\prime}=\sum_{i \in[\alpha]}\left(k-k_{i}^{\prime}\right)$. Let $M_{\alpha+1}=\left(E_{\alpha+1}, \mathcal{I}_{\alpha+1}\right)$ be a uniform matroid over the ground set Fake $(G)$. That is, $M_{\alpha+1}=U_{\tau, k^{\prime}}$. By Propositions 2.1 to Proposition 2.3 we know that $M_{i}$ s are representable over $\mathbb{F}_{p}(X)$, where $p>\max (\tau, 2)$ is a prime number and their representation can be computed in polynomial time. Let $A_{i}$ be the linear representation of $M_{i}$ for all $i \in[\alpha+1]$. Notice that $E_{i} \cap E_{j}=\emptyset$ for all $1 \leq i \neq j \leq \alpha+1$. Let $M$ denote the direct sum $M_{1} \oplus \cdots \oplus M_{\alpha+1}$ with its representation matrix being $A_{M}$. Note that the ground set of $M$ is $\bigcup_{e \in E(G)} \operatorname{Copies}(e)$. Now we define an instance of $\alpha$-MATroid Parity, which is the linear representation $A_{M}$ of $M$ and the partition of ground set into Copies $(e)$, $e \in E(G)$. Notice that for all $e \in E(G), \mid$ Copies $(e) \mid=\alpha$. Also for each $i \in[\alpha], \operatorname{rank}\left(M_{i}\right)=k_{i}^{\prime}$ and $\operatorname{rank}\left(M_{\alpha+1}\right)=k^{\prime}=\sum_{i \in[\alpha]}\left(k-k_{i}^{\prime}\right)$. This implies that $\operatorname{rank}(M)=\alpha k$.

Now Algo-SimFES outputs YES if there is a basis (an independent set of cardinality $\alpha k$ ) of $M$ which is a union of $k$ blocks in $M$ and otherwise outputs No. Algo-SimFES uses the algorithm mentioned in Proposition 2.4 to check whether there is an independent set of $M$, composed of blocks. A pseudocode of Algo-SimFES can be found in Algorithm 1.

- Lemma 3.2. Algo-SimFES is correct.

Proof. Let $\left(G, k, \mathrm{col}: E(G) \rightarrow 2^{[\alpha]}\right)$ be a YES instance of SIM-FES and let $F \subseteq E(G)$, where $|F|=k$ be a solution of $\left(G, k, \mathrm{col}: E(G) \rightarrow 2^{[\alpha]}\right)$. Let $k_{i}=\left|E\left(G_{i}\right)\right|-n+\eta_{i}$, where $\eta_{i}$ is the number of connected components in $G_{i}$, for all $i \in[\alpha]$. For all $i \in[\alpha]$, let $k_{i}^{\prime}=\left|F \cap E\left(G_{i}\right)\right|$. Since $F$ is a solution, $k_{i} \leq k_{i}^{\prime}$ for all $i \in[\alpha]$. This implies that Algo-SimFES will not execute Step 4. Consider the for loop for the choice $\left(k_{1}^{\prime}, \ldots, k_{\alpha}^{\prime}\right)$. We claim that the columns corresponding to $S=\bigcup_{e \in F} \operatorname{Copies}(e)$ form a basis in $M$ and it is union of $k$ blocks. Note that $|S|=\alpha k$ by construction. For all $i \in[\alpha]$, let $F^{i}=\left\{e^{i} \mid e \in F, i \in \operatorname{col}(e)\right\}$, which is subset of ground set of $M_{i}$. By Proposition 3.1, for all $i \in[\alpha], F^{i}$ is a basis for $M_{i}$. This takes care of all the edges in $\cup_{e \in F} \operatorname{Original}(e)$. Now let $S^{*}=S-\cup_{i \in[\alpha]} F^{i}=\cup_{e \in F} \operatorname{Fake}(e)$. Observe that $\left|S^{*}\right|=\sum_{i \in[\alpha]}\left(k-k_{i}^{\prime}\right)=k^{\prime}$. Also, $S^{*}$ is a subset of ground set of $U_{\tau, k^{\prime}}$ and thus is a basis since $\left|S^{*}\right|=k^{\prime}$. Hence $S$ is a basis of $M$. Note that $S$ is the union of blocks corresponding to $e \in F$ and hence is union of $k$ blocks. Therefore, Algo-SimFES will output YES. 
In the reverse direction suppose Algo-SimFES outputs YES. This implies that there is a basis, say $S$, that is the union of $k$ blocks. By construction $S$ corresponds to union of the sets Copies $(e)$ for some $k$ edges in $G$. Let these edges be $F=\left\{e_{1}, \ldots, e_{k}\right\}$. We claim that $F$ is a solution of $\left(G, k, \mathrm{col}: E(G) \rightarrow 2^{[\alpha]}\right)$. Clearly $|F|=k$. Since $S$ is a basis of $M$, for each $i \in[\alpha], B(i)=S \cap\left\{e^{i} \mid e \in E\left(G_{i}\right)\right\}$ is a basis in $M_{i}$. Let $F(i)=\left\{e \mid e^{i} \in B(i)\right\} \subseteq F$. Since $B(i)$ is a basis of $M_{i}$, by Proposition $3.1, G_{i}-F(i)$ is an acyclic graph.

- Lemma 3.3. Algo-SimFES runs in deterministic time $\mathcal{O}\left(2^{\omega k \alpha+\alpha \log k}|V(G)|^{\mathcal{O}(1)}\right)$.

Proof. The for loop runs $(k+1)^{\alpha}$ times. The step 10 uses the algorithm mentioned in Proposition 2.4, which takes time $\mathcal{O}\left(2^{\omega k \alpha}\left\|A_{M}\right\|^{\mathcal{O}(1)}\right)=\mathcal{O}\left(2^{\omega k \alpha}|V(G)|^{\mathcal{O}(1)}\right)$. All other steps in the algorithm takes polynomial time. Thus, the total running time is $\mathcal{O}\left(2^{\omega k \alpha+\alpha \log k}|V(G)|^{\mathcal{O}(1)}\right)$.

Since $\alpha$-MATroid PARITY for $\alpha=2$ can be solved in polynomial time [19] algorithm Algo-SimFES runs in polynomial time for $\alpha=2$. This gives us the following theorem.

- Theorem 3.4. Sim-FES is in FPT and when $\alpha=2$ Sim-FES is in $P$.

\section{Hardness results for Sim-FES}

In this section we show that when $\alpha=3$, SIM-FES is NP-Hard. Furthermore, from our reduction we conclude that it is unlikely that SIM-FES admits a subexponential-time algorithm. We give a reduction from VERTEX COVER (VC) in cubic graphs to the special case of SIM-FES where $\alpha=3$. Let $(G, k)$ be an instance of VC in cubic graphs, which asks whether the graph $G$ has a vertex cover of size at most $k$. We assume without loss of generality that $k \leq|V(G)|$. It is known that $\mathrm{VC}$ in cubic graphs is NP-hard [22] and unless the ETH fails, it cannot be solved in time $\mathcal{O}^{\star}\left(2^{o(|V(G)|+|E(G)|)}\right)^{2}[17]$. Thus, to prove that when $\alpha=3$, it is unlikely that SIM-FES admits a parameterized subexponential time algorithm (an algorithm of running time $\mathcal{O}^{\star}\left(2^{o(k)}\right)$ ), it is sufficient to construct (in polynomial time) an instance of the form $\left(G^{\prime}, k^{\prime}=\mathcal{O}(|V(G)|+|E(G)|), \mathrm{col}^{\prime}: E^{\prime} \rightarrow 2^{[3]}\right)$ of SIM-FES that is equivalent to $(G, k)$. Refer Figure 1 for an illustration of the construction.

To construct $\left(G^{\prime}, k^{\prime}\right.$, col' $\left.^{\prime}: E\left(G^{\prime}\right) \rightarrow 2^{[3]}\right)$, we first construct an instance $(\widehat{G}, \widehat{k})$ of VC in subcubic graphs which is equivalent to $(G, k)$. We set $V(\widehat{G})=V(G) \cup\left(\bigcup_{\{v, u\} \in E(G)}\left\{x_{v, u}, x_{u, v}\right\}\right)$, and $E(\widehat{G})=\left\{\left\{x_{v, u}, x_{u, v}\right\}:\{v, u\} \in E(G)\right\} \cup\left(\bigcup_{\{v, u\} \in E(G)}\left\{\left\{v, x_{v, u}\right\},\left\{u, x_{u, v}\right\}\right\}\right)$. That is, the graph $\widehat{G}$ is obtained from the graph $G$ by subdividing each edge in $E(G)$ twice.

Lemma $4.1(*)$. G has a vertex cover of size $k$ if and only if $\widehat{G}$ has a vertex cover of size $\widehat{k}=k+|E(G)|$

Observe that in $\widehat{G}$ every path between two degree- 3 vertices contains an edge of the form $\left\{x_{v, u}, x_{u, v}\right\}$. Thus, the following procedure results in a partition $\left(M_{1}, M_{2}, M_{3}\right)$ of $E(\widehat{G})$ such that for all $i \in[3],\{v, u\} \in M_{i}$ and $\left\{v^{\prime}, u^{\prime}\right\} \in M_{i} \backslash\{\{v, u\}\}$, it holds that $\{v, u\} \cap\left\{v^{\prime}, u^{\prime}\right\}=\emptyset$. Initially, $M_{1}=M_{2}=M_{3}=\emptyset$. For each degree-3 vertex $v$, let $\{v, x\},\{v, y\}$ and $\{v, z\}$ be the edges containing $v$. We insert $\{v, x\}$ into $M_{1},\{v, y\}$ into $M_{2}$, and $\{v, z\}$ into $M_{3}$ (the choice of which edge is inserted into which set is arbitrary). Finally, we insert each edge of the form $\left\{x_{v, u}, x_{u, v}\right\}$ into a set $M_{i}$ that contains neither $\left\{v, x_{v, u}\right\}$ nor $\left\{u, x_{u, v}\right\}$.

We are now ready to construct the instance $\left(G^{\prime}, k^{\prime}\right.$, col $\left.^{\prime}: E\left(G^{\prime}\right) \rightarrow 2^{[3]}\right)$. Let $V\left(G^{\prime}\right)=$ $V(\widehat{G}) \cup V^{\star}$, where $V^{\star}=\left\{v^{\star}: v \in V(\widehat{G})\right\}$ contains a copy $v^{\star}$ of each vertex $v$ in $V(\widehat{G})$. The

$2 \mathcal{O}^{\star}$ notation suppresses polynomial factors in the running-time expression. 


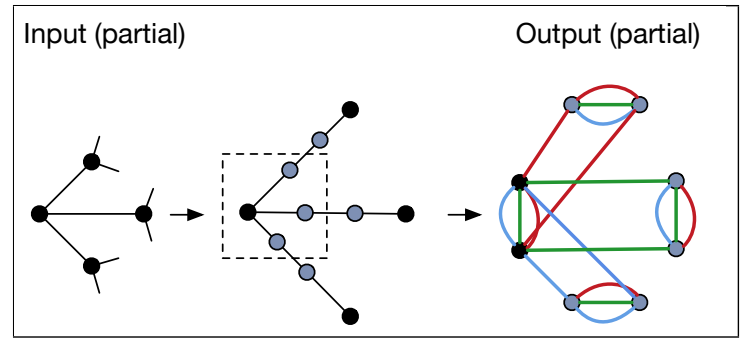

Figure 1 The construction given in the proof of Theorem 4.3.

set $E\left(G^{\prime}\right)$ and coloring col' are constructed as follows. For each vertex $v \in V(\widehat{G})$, add an edge $\left\{v, v^{\star}\right\}$ into $E\left(G^{\prime}\right)$ and its color-set is $\{1,2,3\}$. For each $i \in[3]$ and for each $\{v, u\} \in M_{i}$, add the edges $\{v, u\}$ and $\left\{v^{\star}, u^{\star}\right\}$ into $E\left(G^{\prime}\right)$ and its color-set is $\{i\}$. We set $k^{\prime}=\widehat{k}$. Clearly, the instance $\left(G^{\prime}, k^{\prime}\right.$, col' $\left.^{\prime}: E\left(G^{\prime}\right) \rightarrow 2^{[3]}\right)$ can be constructed in polynomial time, and it holds that $k^{\prime}=\mathcal{O}(|V(G)|+|E(G)|)$.

Lemma 4.2 proves that $(\widehat{G}, \widehat{k})$ is a YES instance of VC if and only if $\left(G^{\prime}, k^{\prime}, \mathrm{col}^{\prime}: E\left(G^{\prime}\right) \rightarrow\right.$ $\left.2^{[3]}\right)$ is a YES instance of Sim-FES. Observe that because of the above mentioned property of the partition $\left(M_{1}, M_{2}, M_{3}\right)$ of $E(\widehat{G})$, we ensure that in $G^{\prime}$, no vertex participates in two (or more) monochromatic cycles that have the same color. By construction, each monochromatic cycle in $G^{\prime}$ is of the form $v-v^{\star}-u^{\star}-u-v$, where $\{v, u\} \in E(\widehat{G})$, and for each edge $\{v, u\} \in E\left(G^{\prime}\right)$, where either $v, u \in V(\widehat{G})$ or $v, u \in V^{\star}, G^{\prime}$ contains exactly one monochromatic cycle of this form.

- Lemma $4.2(*) .(\widehat{G}, \widehat{k})$ is a YES instance of $V C$ if and only if $\left(G^{\prime}, k^{\prime}, \operatorname{col}^{\prime}: E\left(G^{\prime}\right) \rightarrow 2^{[3]}\right)$ is a YES instance of SIM-FES.

We get the following theorem and its proof follows from Lemma 4.1 and Lemma 4.2.

- Theorem 4.3. SIM-FES where $\alpha=3$ is NP-hard. Furthermore, unless the Exponential Time Hypothesis (ETH) fails, Sim-FES when $\alpha=3$ cannot be solved in time $\mathcal{O}^{*}\left(2^{o(k)}\right)$.

\section{Tight Lower Bounds for Simultaneous Feedback Edge Set}

We show that SIM-FES parameterized by $k$ is $W[2]$ hard when $\alpha=\mathcal{O}(|V(G)|)$ and $W[1]$ hard when $\alpha=\mathcal{O}(\log (|V(G)|))$. Our reductions follow the approach of Agrawal et al. [1].

W[2] Hardness of Sim-FES when $\alpha=\mathcal{O}(|\boldsymbol{V}(G)|)$. We give a reduction from HiTTING Set (HS) to Sim-FES where $\alpha=\mathcal{O}(|V(G)|)$. Let $\left(U=\left\{u_{1}, u_{2}, \ldots, u_{|U|}\right\}, \mathcal{F}=\right.$ $\left.\left\{F_{1}, F_{2}, \ldots, F_{|\mathcal{F}|}\right\}, k\right)$ be an instance of HS, where $\mathcal{F} \subseteq 2^{U}$, which asks whether there exists a subset $S \subseteq U$ of size at most $k$ such that for all $F \in \mathcal{F}, S \cap F \neq \emptyset$. It is known that HS parameterized by $k$ is W[2]-hard (see, e.g., [9]). Thus, to prove the result, it is sufficient to construct (in polynomial time) an instance of the form $\left(G, k\right.$, col $\left.: E(G) \rightarrow 2^{[\alpha]}\right)$ of Sim-FES that is equivalent to $(U, \mathcal{F}, k)$, where $\alpha=\mathcal{O}(|V(G)|)$. We construct a graph $G$ such that $V(G)=\mathcal{O}(|U||\mathcal{F}|)$ and the number of colors used will be $\alpha=|\mathcal{F}|$. The intuitive idea is to have one edge per element in the universe which is colored with all the indices of sets in the family $\mathcal{F}$ that contains the element and for each $F_{i} \in \mathcal{F}$ creating a unique monochromatic cycle with color $i$ which passes through all the edges corresponding to the elements it contain. We explain the reduction formally in the next paragraph. 
Without loss of generality we assume that each set in $\mathcal{F}$ contains at least two elements from $U$. The instance $\left(G, k, \mathrm{col}: E(G) \rightarrow 2^{[\alpha]}\right)$ is constructed as follows. Initially, $V(G)=$ $E(G)=\emptyset$. For each element $u_{i} \in U$, insert two new vertices into $V(G), v_{i}$ and $w_{i}$, add the edge $\left\{v_{i}, w_{i}\right\}$ into $E(G)$ and let $\left\{j \mid F_{j} \in \mathcal{F}, u_{i} \in F_{j}\right\}$ be its color-set. Now, for all $1 \leq i<j \leq|U|$ and for all $1 \leq t \leq|\mathcal{F}|$ such that $u_{i}, u_{j} \in F_{t}$ and $\left\{u_{i+1}, \ldots, u_{j-1}\right\} \cap F_{t}=\emptyset$, perform the following operation: add a new vertex into $V(G), s_{i, j, t}$, add the edges $\left\{w_{i}, s_{i, j, t}\right\}$ and $\left\{s_{i, j, t}, v_{j}\right\}$ into $E(G)$ and let their color-set be $\{t\}$. Moreover, for each $1 \leq t \leq|\mathcal{F}|$, let $u_{i}$ and $u_{j}$ be the elements with the largest and smallest index contained in $F_{t}$, respectively, and perform the following operation: add a new vertex into $V(G), s_{i, j, t}$, add the edges $\left\{w_{i}, s_{i, j, t}\right\}$ and $\left\{s_{i, j, t}, v_{j}\right\}$ into $E(G)$, and let their color-set be $\{t\}$. Observe that $|V(G)|=\mathcal{O}(|U||\mathcal{F}|)$ and that $\alpha=|\mathcal{F}|$. Therefore, $\alpha=\mathcal{O}(|V(G)|)$. It remains to show that the instances $(G, k, \mathrm{col})$ and $(U, \mathcal{F}, k)$ are equivalent. By construction, each monochromatic cycle in $G$ is of the form $v_{i_{1}}-w_{i_{1}}-s_{i_{1}, i_{2}, t}-v_{i_{2}}-w_{i_{2}}-s_{i_{2}, i_{3}, t}-\cdots-v_{i_{\left|F_{t}\right|}}-w_{i_{\left|F_{t}\right|}}-s_{i_{\mid F_{t}, i_{1}, t}}-v_{i_{1}}$, where $\left\{u_{i_{1}}, u_{i_{2}}, \ldots, u_{i_{\left|F_{t}\right|}}\right\}=F_{t} \in \mathcal{F}$, and for each set $F_{t} \in \mathcal{F}, G$ contains exactly one such monochromatic cycle.

- Lemma $5.1(*) .(U, \mathcal{F}, k)$ is a YES instance of HS if and only if $\left(G, k, c o l: E(G) \rightarrow 2^{[\alpha]}\right)$ is a YES instance of SIM-FES.

Theorem 5.2. SIM-FES parameterized by $k$, when $\alpha=\mathcal{O}(|V(G)|)$, is $W[2]$-hard.

W[1] Hardness of Sim-FES when $\alpha=\mathcal{O}(\log |\boldsymbol{V}(G)|)$. We modify the reduction given in the proof of Theorem 5.2 to show that when $\alpha=\mathcal{O}(\log |V(G)|)$, Sim-FES is W[1]-hard with respect to the parameter $k$. This result implies that the dependency on $\alpha$ of our $\mathcal{O}\left(\left(2^{\mathcal{O}(\alpha)}\right)^{k} n^{\mathcal{O}(1)}\right)$-time algorithm for SIM-FES is optimal in the sense that it is unlikely that there exists an $\mathcal{O}\left(\left(2^{o(\alpha)}\right)^{k} n^{\mathcal{O}(1)}\right)$-time algorithm for this problem.

We give a reduction from a variant of HS, called Partitioned Hitting Set (PHS), to Sim-FES where $\alpha=\mathcal{O}(\log |V(G)|)$. The input of PHS consists of a universe $U$, a collection $\mathcal{F}=\left\{F_{1}, F_{2}, \ldots, F_{|\mathcal{F}|}\right\}$, where each $F_{i}$ is a family of disjoint subsets of $U$, and a parameter $k$. The goal is to decide the existence of a subset $S \subseteq U$ of size at most $k$ such that for all $f \in$ $\left(\bigcup_{F \in \mathcal{F}} F\right), S \cap f \neq \emptyset$. It is known that the special case of PHS where $|\mathcal{F}|=\mathcal{O}(\log (|U||(\bigcup \mathcal{F})|))$ is W[1]-hard when parameterized by $k$ (see, e.g., [1]). Thus, to prove the theorem, it is sufficient to construct (in polynomial time) an instance of the form $\left(G, k\right.$, col : $\left.E(G) \rightarrow 2^{[\alpha]}\right)$ of SIM-FES that is equivalent to $(U, \mathcal{F}, k)$, where $\alpha=\mathcal{O}(\log |V(G)|)$. The construction of the graph $G$ is exactly similar to the one in Theorem 5.2. But instead of creating a unique monochromatic cycle with a color $i$ for each $f_{i} \in \bigcup \mathcal{F}$, for each $F_{i} \in \mathcal{F}$ we create $\left|F_{i}\right|$ vertex disjoint cycles of same color $i$. Since for each $F \in \mathcal{F}$ the sets in $F$ are pairwise disjoint, guarantees the correctness. Formal description of the reduction is given below.

Without loss of generality we assume that each set in $\bigcup_{F \in \mathcal{F}} F$ contains at least two elements from $U$. The instance $\left(G, k, \mathrm{col}: E(G) \rightarrow 2^{[\alpha]}\right)$ is constructed as follows. Initially, $V(G)=E(G)=\emptyset$. For each element $u_{i} \in U$, insert two new vertices $v_{i}$ and $w_{i}$ into $V(G)$, and add the edge $\left\{v_{i}, w_{i}\right\}$ into $E(G)$ with its color-set being $\left\{j: F_{j} \in \mathcal{F}, u_{i} \in\left(\bigcup F_{j}\right)\right\}$. Now, for all $1 \leq i<j \leq|U|$ and for all $1 \leq t \leq|\mathcal{F}|$ such that there exists $f \in F_{t}$ satisfying $u_{i}, u_{j} \in f$ and $\left\{u_{i+1}, \ldots, u_{j-1}\right\} \cap f=\emptyset$, perform the following operation: add a new vertex $s_{i, j, t}$ into $V(G)$, add the edges $\left\{w_{i}, s_{i, j, t}\right\}$ and $\left\{s_{i, j, t}, v_{j}\right\}$ into $E(G)$ with both of its color-set being $\{t\}$. Moreover, for each $1 \leq t \leq|\mathcal{F}|$ and $f \in F_{t}$, let $u_{i}$ and $u_{j}$ be the elements with the largest and smallest index contained in $f$, respectively, we perform the following operation: add a new vertex into $V(G), s_{i, j, t}$, add the edges $\left\{w_{i}, s_{i, j, t}\right\}$ and $\left\{s_{i, j, t}, v_{j}\right\}$ into $E(G)$, and let their color-set be $\{t\}$. Observe that $|V(G)|=\mathcal{O}(|U||(\bigcup \mathcal{F})|)$ and that $\alpha=|\mathcal{F}|$. 
Since $|\mathcal{F}|=\mathcal{O}(\log (|U||(\bigcup \mathcal{F})|))$, we have that $\alpha=\mathcal{O}(\log |V(G)|)$. Since the sets in each family $F_{i}$ are disjoint, the construction implies that each monochromatic cycle in $G$ is of the form $v_{i_{1}}-w_{i_{1}}-s_{i_{1}, i_{2}, t}-v_{i_{2}}-w_{i_{2}}-s_{i_{2}, i_{3}, t}-\cdots-v_{i_{|f|}}-w_{i_{|f|}}-s_{i_{|f|}, i_{1}, t}-v_{i_{1}}$, where $\left\{u_{i_{1}}, u_{i_{2}}, \ldots, u_{i_{\left|F_{t}\right|}}\right\}=f$ for a set $f \in F_{t} \in \mathcal{F}$, and for each set $f \in F_{t} \in \mathcal{F}, G$ contains a monochromatic cycle of this form. By using the arguments similar to one in the proof of Lemma 5.1, we get that the instances $\left(G, k\right.$, col : $\left.E(G) \rightarrow 2^{[\alpha]}\right)$ and $(U, \mathcal{F}, k)$ are equivalent. Hence we get the following theorem.

Theorem 5.3. Sim-FES parameterized by $k$, when $\alpha=\mathcal{O}(\log |V(G)|)$ is $W[1]$-hard.

\section{Kernel for Simultaneous Feedback Edge Set}

In this section we give a kernel for SIM-FES with $\mathcal{O}\left((k \alpha)^{\mathcal{O}(\alpha)}\right)$ vertices. We start by applying preprocessing rules similar in spirit to the ones used to obtain a kernel for FEEDBACK VERTEX SET, but it requires subtle differences due to the fact that we handle a problem where edges rather than vertices are deleted, as well as the fact that the edges are colored (in particular, each edge in SIm-FES has a color-set, while each vertex in SIm-FVS is uncolored). We obtain an approximate solution by computing a spanning tree per color. We rely on the approximate solution to bound the number of vertices whose degree in certain subgraphs of $G$ is not equal to 2 . Then, the number of the remaining vertices is bounded by adapting the "interception"-based approach of Agrawal et al. [1] to a form relevant to Sim-FES.

Let $\left(G, k, \mathrm{col}: E(G) \rightarrow 2^{[\alpha]}\right)$ be an instance of SIM-FES. For each color $i \in[\alpha]$ recall $G_{i}$ is the graph consisting of the vertex-set $V(G)$ and the edge-set $E\left(G_{i}\right)$ includes every edge in $E(G)$ whose color-set contains the color $i$. It is easy to verify that the following rules are correct when applied exhaustively in the order in which they are listed. We note that the resulting instance can contain multiple edges.

- Reduction Rule 1: If $k<0$, return that $\left(G, k, \mathrm{col}: E(G) \rightarrow 2^{[\alpha]}\right)$ is a No instance.

- Reduction Rule 2: If for all $i \in[\alpha], G_{i}$ is acyclic, return that $\left(G, k\right.$, col $\left.: E(G) \rightarrow 2^{[\alpha]}\right)$ is a YEs instance.

- Reduction Rule 3: If there is a self-loop at a vertex $v \in V(G)$, then remove $v$ from $G$ and decrement $k$ by 1.

- Reduction Rule 4: If there exists an isolated vertex in $G$, then remove it.

- Reduction Rule 5: If there exists $i \in[\alpha]$ and an edge whose color-set contains $i$ but it does not participate in any cycle in $G_{i}$, remove $i$ from its color-set. If the color-set becomes empty, remove the edge.

- Reduction Rule 6: If there exists $i \in[\alpha]$ and a vertex $v$ of degree exactly two in $G$, remove $v$ and connect its two neighbors by an edge whose color-set is the same as the color-set of the two edges incident to $v$ (we prove in Lemma 6.1 that the color set of two edges are same).

- Lemma $6.1(*)$. Reduction rule 6 is safe.

We apply Reduction Rule 1 to 6 exhaustively (in that order). The safeness of Reduction Rules 1 to 5 are easy to see. Lemma 6.1 proves the safeness Reduction Rule 6 . After this, we follow the approach similar to that in [1] to bound the size of the instance. This gives the following theorem.

- Theorem 6.2 (*). SIM-FES admits a kernel with $(k \alpha)^{\mathcal{O}(\alpha)}$ vertices. 


\section{Maximum Simultaneous Acyclic Subgraph}

In this section we design an algorithm for Maximum Simultaneous Acyclic Subgraph. Let $\left(G, q\right.$, col : $\left.E(G) \rightarrow 2^{[\alpha]}\right)$ be an input to MAX-SIm-SubGraph. A set $F \subseteq E(G)$ such that for all $i \in[\alpha], G\left[F_{i}\right]$ is acyclic is called simultaneous forest. Here, $F_{i}=\{e \in F \mid i \in \operatorname{col}(e)\}$, denotes the subset of edges of $F$ which has the integer $i$ in its image when the function col is applied to it. We will solve MAX-SIM-SUBGRAPH by reducing to an equivalent instance of the $\alpha$-MATroID PARITY problem and then using the algorithm for the same.

We start by giving a construction that reduces the MAX-SIM-SUBGRAPH to $\alpha$-MATROID PARITy. Let $\left(G, q, \mathrm{col}: E(G) \rightarrow 2^{[\alpha]}\right)$ be an input to MAX-Sim-SubGraph. Given, $(G, q$, col : $\left.E(G) \rightarrow 2^{[\alpha]}\right)$, for $i \in[\alpha]$, recall that by $G_{i}$ we denote the graph with the vertex set $V\left(G_{i}\right)=V(G)$ and the edge set $E\left(G_{i}\right)=\left\{e^{i} \mid e \in E(G)\right.$ and $\left.i \in \operatorname{col}(e)\right\}$. For each edge $e \in E(G)$, we will have its distinct copy in $G_{i}$ if $i \in \operatorname{col}(e)$. Thus, for each edge $e \in E(G)$, by Original $(e)$ we denote the set of edges $\left\{e^{j} \mid j \in \operatorname{col}(e)\right\}$. On the other hand for each edge $e \in E(G)$, by Fake $(e)$ we denote the set of edges $\left\{e^{j} \mid j \in[\alpha]-\operatorname{col}(e)\right\}$. Finally, for each edge $e \in E(G)$, by Copies $(e)$ we denote the set $\operatorname{Original}(e) \cup \operatorname{Fake}(e)$. Let $M_{i}=\left(E_{i}, \mathcal{I}_{i}\right)$ denote the graphic matroid on $G_{i}$. That is, edges of $G_{i}$ forms the universe $E_{i}$ and $\mathcal{I}_{i}$ contains, $S \subseteq E\left(G_{i}\right)$ such that $G_{i}[S]$ forms a forest. By Proposition 2.2 we know that graphic matroids are representable over any field and given a graph $G$ one can find the corresponding representation matrix in time polynomial in $|V(G)|$. Let $A_{i}$ denote the linear representation of $M_{i}$. That is, $A_{i}$ is a matrix over $\mathbb{F}_{2}$, where the set of columns of $A_{i}$ are denoted by $E\left(G_{i}\right)$. In particular, $A_{i}$ has dimension $d \times\left|E\left(G_{i}\right)\right|$, where $d=\operatorname{rank}\left(M_{i}\right)$. A set $X \subseteq E\left(G_{i}\right)$ is independent (that is $X \in \mathcal{I}_{i}$ ) if and only if the corresponding columns are linearly independent over $\mathbb{F}_{2}$. Let Fake $(G)$ denote the set of edges in $\bigcup_{e \in E(G)}$ Fake $(e)$. Furthermore, let $\tau=|\operatorname{Fake}(G)|=\sum_{e \in E(G)}|\operatorname{Fake}(e)|$. Let $M_{\alpha+1}$ be the uniform matroid over Fake $(G)$ of rank $\tau$. That is, $E_{\alpha+1}=\operatorname{Fake}(G)$ and $M_{\alpha+1}=U_{\tau, \tau}$. Let $I_{\tau}$ denote the identity matrix of dimension $\tau \times \tau$. Observe that, $A_{\alpha+1}=I_{\tau}$ denotes the linear representation of $M_{\alpha+1}$ over $\mathbb{F}_{2}$. Notice that $E_{i} \cap E_{j}=\emptyset$ for all $1 \leq i \neq j \leq \alpha+1$. Let $M$ denote the direct sum of $M_{1} \oplus \cdots \oplus M_{\alpha+1}$ with its representation matrix being $A_{M}$.

Now we are ready to define an instance of $\alpha$-Matroid PARITY. The ground set is the columns of $A_{M}$, which is indexed by edges in $\bigcup_{e \in E(G)} \operatorname{Copies}(e)$. Furthermore, the ground set is partitioned into Copies $(e), e \in E(G)$, which are called blocks. The main technical lemma of this section on which the whole algorithm is based is the following.

- Lemma $7.1(*)$. Let $\left(G, q, \mathrm{col}: E(G) \rightarrow 2^{[\alpha]}\right)$ be an instance of MAX-Sim-SubGraPH. Then $G$ has a simultaneous forest of size $q$ if and only if $\left(A_{M}, \biguplus_{e \in E(G)} \operatorname{Copies}(e), q\right)$ is a YES instance of $\alpha$-MATroid PARITY. Furthermore, given $\left(G, q, \mathrm{col}: E(G) \rightarrow 2^{[\alpha]}\right)$ we can obtain an instance $\left(A_{M}, \biguplus_{e \in E(G)}\right.$ Copies $\left.(e), q\right)$ in polynomial time.

We will use the polynomial time reduction provided in Lemma 7.1 to get the desired FPT algorithm for MAX-Sim-SubGRAPH. Towards this will use the following FPT result regarding $\alpha$-MAtroid PARITy for our FPT as well as for an exact exponential time algorithm.

Given an instance $\left(G, q\right.$, col $\left.: E(G) \rightarrow 2^{[\alpha]}\right)$ of MAX-SIM-SubGrAPH we first apply Lemma 7.1 and obtain an instance $\left(A_{M}, \biguplus_{e \in E(G)} \operatorname{Copies}(e), q\right)$ of $\alpha$-MAtroid PARITy and then apply Proposition 2.4 to obtain the following result.

- Theorem 7.2. MAX-Sim-SubGraph can be solved in time $\mathcal{O}\left(2^{\omega q \alpha}|V(G)|^{\mathcal{O}(1)}\right)$.

Let $\left(G, q, \mathrm{col}: E(G) \rightarrow 2^{[\alpha]}\right)$ be an instance of MAX-Sim-SubGrAPH. Observe that $q$ is upper bounded by $\alpha(|V(G)|-1)$. Thus, as a corollary to Theorem 7.2 we get an 
exact algorithm for finding the largest sized simultaneous acyclic subgraph, running in time $\mathcal{O}\left(2^{\omega n \alpha^{2}}|V(G)|^{\mathcal{O}(1)}\right)$.

\section{References}

1 A. Agrawal, D. Lokshtanov, A. E. Mouawad, and S. Saurabh. Simultaneous feedback vertex set: A parameterized perspective. In 33rd Symposium on Theoretical Aspects of Computer Science, STACS, pages 7:1-7:15, 2016.

2 Akanksha Agrawal, Fahad Panolan, Saket Saurabh, and Meirav Zehavi. Simultaneous feedback edge set: A parameterized perspective. CoRR, abs/1611.07701, 2016. URL: https://arxiv.org/abs/1611.07701.

3 Noga Alon and Timothy H. Marshall. Homomorphisms of edge-colored graphs and coxeter groups. Journal of Algebraic Combinatorics, 8(1):5-13, 1998.

4 Vineet Bafna, Piotr Berman, and Toshihiro Fujito. A 2-approximation algorithm for the undirected feedback vertex set problem. SIAM Journal on Discrete Mathematics, 12(3):289297, September 1999.

5 József Balogh, János Barát, Dániel Gerbner, András Gyárfás, and Gábor N. Sárközy. Partitioning 2-edge-colored graphs by monochromatic paths and cycles. Combinatorica, 34(5):507-526, 2014.

6 Jørgen Bang-Jensen and Gregory Gutin. Alternating cycles and paths in edge-coloured multigraphs: a survey. Discrete Mathematics, 165:39-60, 1997.

7 Leizhen Cai and Junjie Ye. Dual connectedness of edge-bicolored graphs and beyond. In Mathematical Foundations of Computer Science, MFCS, volume 8635, pages 141-152, 2014.

8 W.S. Chou, Yannis Manoussakis, Olga Megalakaki, M. Spyratos, and Zs. Tuza. Paths through fixed vertices in edge-colored graphs. Mathématiques et sciences humaines, 127:4958, 1994.

9 M. Cygan, F. V. Fomin, L. Kowalik, D. Lokshtanov, D. Marx, M. Pilipczuk, M. Pilipczuk, and S. Saurabh. Parameterized algorithms. Springer, 2015.

10 Marek Cygan, Jesper Nederlof, Marcin Pilipczuk, Michał Pilipczuk, Joham M. M. van Rooij, and Jakub Onufry Wojtaszczyk. Solving connectivity problems parameterized by treewidth in single exponential time. In Foundations of Computer Science (FOCS), IEEE 52nd Annual Symposium, pages 150-159, 2011.

11 Reinhard Diestel. Graph Theory, 4th Edition, volume 173 of Graduate texts in mathematics. Springer, 2012.

12 Fedor V. Fomin, Serge Gaspers, Daniel Lokshtanov, and Saket Saurabh. Exact algorithms via monotone local search. In Proceedings of the 48th Annual ACM SIGACT Symposium on Theory of Computing, STOC, pages 764-775, 2016.

13 M.R. Garey and D.S. Johnson. Computers and intractability: A guide to the theory of NP-completeness. W. H. Freeman \& Co., 1979.

14 Russell Impagliazzo, Ramamohan Paturi, and Francis Zane. Which problems have strongly exponential complexity? Journal of Computer and System Sciences, 63(4):512-530, 2001.

15 Mikio Kano and Xueliang Li. Monochromatic and heterochromatic subgraphs in edgecolored graphs-a survey. Graphs and Combinatorics, 24(4):237-263, 2008.

16 Tomasz Kociumaka and Marcin Pilipczuk. Faster deterministic feedback vertex set. Information Processing Letters, 114(10):556-560, 2014.

17 Christian Komusiewicz. Tight running time lower bounds for vertex deletion problems. arXiv preprint arXiv:1511.05449, 2015.

18 Daniel Lokshtanov, Pranabendu Misra, Fahad Panolan, and Saket Saurabh. Deterministic truncation of linear matroids. In Automata, Languages, and Programming, pages 922-934. Springer, 2015. 
19 László Lovász. Matroid matching and some applications. Journal of Combinatorial Theory, Series B, 28(2):208-236, 1980.

20 Yannis Manoussakis. Alternating paths in edge-colored complete graphs. Discrete Applied Mathematics, 56(2):297-309, 1995.

21 Dániel Marx. A parameterized view on matroid optimization problems. Theoretical Computer Science, 410(44):4471-4479, October 2009.

22 Bojan Mohar. Face covers and the genus problem for apex graphs. Journal of Combinatorial Theory, Series B, 82(1):102-117, 2001.

23 James G Oxley. Matroid theory, volume 3. Oxford University Press, USA, 2006.

24 Stéphan Thomassé. A $4 k^{2}$ kernel for feedback vertex set. ACM Transactions on Algorithms, (TALG), 6(2):32:1-32:8, 2010.

25 Virginia Vassilevska Williams. Multiplying matrices faster than coppersmith-winograd. In Proceedings of the 44th annual ACM symposium on Theory of computing, pages 887-898, 2012. 